\title{
Effective Kinetic Phase Diagrams
}

\author{
Jan H. Los* \\ IMM Laboratory of Solid State Chemistry, Radboud University Nijmegen, Toernooiveld, \\ 6525 ED Nijmegen, The Netherlands
}

\section{Marija Matovic \\ Chemical Thermodynamics Group, Debye Institute, Utrecht University, Padualaan 8, 3584 CH Utrecht, The Netherlands}

Received: March 17, 2005; In Final Form: May 25, 2005

\begin{abstract}
The composition of a solid solution that is growing at conditions well away from equilibrium is not prescribed by equilibrium thermodynamics, but is determined kinetically. It depends both on the surface kinetics and on the transport of mass and heat to and away from the solidification front. In previous work, we have formulated a model for the kinetic or nonequilibrium segregation taking place at the solidification front enabling the construction of kinetic phase diagrams, which gives the growth composition of a solid solution as a function of the liquid composition and undercooling at the surface. In the present work, we extend this model to include both mass and heat transport, giving rise to effective kinetic phase diagrams. An overview of the tendencies in the calculated effective kinetic phase diagrams is given by scanning a large part of the parameter space, covering different types of materials, including metals, semiconductors, and molecular systems. We find striking and characteric differences in the relative contribution of the various processes to the effective segregation. For molecular mixtures, interfacial undercooling and heat transport limitation can be expected to be much more important than for metal and semiconductor mixtures where mass transport limitation is dominant.
\end{abstract}

\section{Introduction}

Substitutional solid solutions are solid phases in which the atomic or molecular positions belonging to a perfect periodic structure are occupied by different species in a random way. ${ }^{1}$ They represent a quite important class of materials due their intrinsic possibility of tuning their properties by varying the composition. Also, knowledge of the miscibility of different species in the solid phase and, in particular, the role of the kinetics during the growth process on the mixing is quite relevant for separation techniques with applications in the food and pharmaceutical industries. ${ }^{2}$

The equilibrium state of a mixed liquid/solid system, containing a liquid and a solid fraction, is completely fixed thermodynamically once the pure component properties and the excess Gibbs free energy, usually expressed in terms of a number of excess parameters, are known. ${ }^{3-5}$ However, crystallization is a kinetic process, and the growth composition, i.e., the instantaneous composition of the solid phase being formed at the surface at large undercooling may deviate considerably from the composition according to the equilibrium phase diagram. Under batch conditions the composition of the mother phase will change, and thus also the growth composition will change during the crystallization, leading to composition gradients in the solid phase. ${ }^{6,7}$ These gradients may stay for very long periods due to the very low diffusion rate in solid phases, especially in molecular solid phases. Hence, in such mixed systems, the equilibrium state will hardly ever be reached and the system will stay in a metastable state. A prediction of this nonequilibrium state requires a kinetic modeling of the crystallization process, with first of all a description of the kinetic segregation

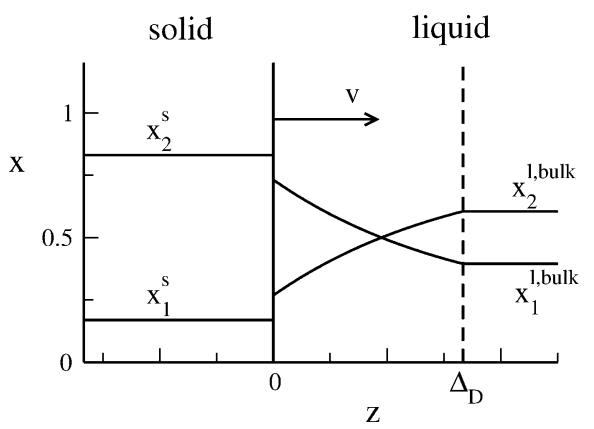

Figure 1. Schematic illustration of mass transport limitation.

at the solidification front as a function of the interfacial undercooling and composition of the liquid phase. However, the segregation at the growth front will induce gradients in the liquid phase, both in the concentrations of the components involved and in the temperature. Components that are build preferentially in the solid phase will be depleted in the liquid phase directly at the interface, whereas rejected components will accumulate. This is illustrated in Figure 1 for a binary system. The changes in the liquid concentrations at the interface will induce diffusional fluxes of the components between the interface and the bulk liquid phase, but it will also affect the interfacial segregation. Eventually, a steady state is reached, where the incorporation rate at the interface is balanced by the rate of transport for each component. Similarly, the heat of crystallization produced at the interface, giving rise to a temperature gradient between the interface and the bulk fluid phase, will be balanced by the diffusion of heat away from the interface. As for mass transport limitation, also heat transport 
limitation will affect the interfacial segregation, which depends on the temperature at the interface. (The term limitation refers to the finiteness of the diffusion rates causing the gradients.) Thus, surface integration, mass transport and heat transport are all coupled.

In the past, kinetic segregation during the growth of a solid solution has been studied theoretically using a lattice Monte Carlo (MC) simulation model, ${ }^{8,9}$ based on the Kossel model, ${ }^{10,11}$ or kinetic equations. ${ }^{12,13}$ Mostly, the latter models are continuum descriptions based on the MC model. These works focus on the temperature dependence of the interfacial segregation without taking into account the effect of diffusion limitation. Opposite to that is the model of Burton, Prim, and Slichter (BPS ${ }^{14}$ which assumes near equilibrium at the growing surface, i.e., the interfacial segregation is equal to the equilibrium segregation for the liquid composition at the interface. It takes into account mass transport limitation, but heat transport limitation is neglected. This has led to the famous BPS equation, which expresses the effective segregation as a function of the crystal growth velocity. Other work on kinetic segregation ${ }^{15-19}$ was focused on the low concentration limit in order to explain the observed high incorporation of dopants into silicon after laser melting. ${ }^{20-22}$ These models, which integrate interfacial segregation and mass transport in a semiempirical way, apply to regular dilute solutions and more or less successfully describe the observed nonequilibrium segregation as a function of the crystal growth velocity ${ }^{18,23-25}$ after fitting one or a few parameter(s). Recently, detailed studies of the interfacial kinetic segregation, based on the Kossel model, have appeared. ${ }^{26-28}$ In these works, which apply to ideal, dilute solutions, relations for effective segregation coefficients for different types of sites (i.e., kink, step, terrace, and bulk sites) are derived.

In the present work we propose a theoretical model for the effective kinetic segregation during growth of a binary solid solution which integrates the interfacial kinetic segregation, mass transport limitation, and heat transport limitation. To our knowledge, such a model that couples all three processes has not been introduced previously. Furthermore, the model is not restricted to dilute and ideal or regular solutions, but applies to a binary system of any composition and for any crystal lattice with known equilibrium mixing properties for the liquid and solid phases. In our model the interfacial segregation is described by the linear kinetic segregation (LKS) model, ${ }^{29,6}$ which is based on nonequilibrium thermodynamics and a linear dependence of the growth rates of each of the individual components on their absolute supersaturation. In this model, the fluxes from one phase to the other are related by the principle of microscopic reversibility, which, strictly speaking, is only valid for nearequilibrium. However, in MC simulations of the growth of a binary crystal, based on the Kossel model we find that the trends of the LKS model are in qualitative agreement with those of the MC simulation. Basically, for increasing undercooling the composition of the crystal tends toward, and eventually becomes equal to, the composition of the liquid phase. More in detail, comparing the segregation as a function of the undercooling, there are quantitative differences between the results from the LKS model and those of the MC simulations. However, these are relatively small as long as the various bond energies determining the surface kinetics in the MC model are not too much different. In practice, this is often the case for isomorphous constituents forming solid solutions. A detailed confrontation between the LKS model and the MC simulation model for a binary system of arbitrary composition will be given elsewhere. ${ }^{30}$

As we will show, a coupled description of the interfacial segregation and transport effects is particularly relevant for molecular systems, which usually have relative large $\phi /\left(k_{\mathrm{B}} \tilde{T}_{\mathrm{m}}\right)$ values, where $\phi$ is the bond energy and $\tilde{T}_{\mathrm{m}}$ is the average melting temperature. According to the LKS model, as well as the MC model, in such cases the interfacial segregation can deviate considerably from the equilibrium segregation already at moderate undercoolings. When mass and heat transport take place relatively fast, eventually stimulated by stirring, such undercoolings can easily occur at the interface.

For studying the interfacial segregation experimentally, the coupling with transport effects is quite important for the interpretation of the experimental data. Usually only the bulk properties are well-known, whereas the actual conditions at the surface are much more difficult to access experimentally. Theory provides additional, useful tools for a correct interpretation.

We will refer to our model as the linear effective kinetic segregation (LEKS) model. It is described in detail in section 2. In section 3, effective kinetic phase diagrams (EKPDs), calculated according to the LEKS model, are presented and discussed. We conclude with a summary and discussion in section 4 .

\section{Theory}

As already mentioned, the effective segregation during the growth of mixed crystals requires a coupled description of the interfacial segregation, mass transport, and heat transport. In our description of this problem, the first part, i.e., the interfacial segregation, is based on the LKS model. Although the LKS model has been decribed elsewhere, ${ }^{29,6}$ we have decided to include a short recapitulation of it, which we think enhances the readability of the present work significantly and at the same time gives us the opportunity to introduce the terminology. Moreover, we have adopted a different, more general excess energy model, as described below.

2.1. Linear Kinetic Segregation (LKS) Model. Considering the growth of a binary, mixed crystal from a liquid mother phase, nonequilibrium thermodynamics tells us that the flux of a component $i$ from the liquid to the solid phase, $J_{i}^{+}$, and the reverse flux, $J_{i}^{-}$, are related by

$$
\frac{J_{i}^{+}}{J_{i}^{-}}=\exp \left(\frac{\Delta \mu_{i}^{\text {surf }}}{R T^{\text {surf }}}\right)
$$

where $T^{\text {surf }}$ is the temperature at the surface and $\Delta \mu_{i}^{\text {surf }}=\mu_{i}^{1, \text { surf }}$ $-\mu_{i}^{\mathrm{s}, \mathrm{gr}}$ is the difference between the chemical potential of component $i$ in the liquid phase at the surface, $\mu_{i}^{\text {l,surf }}$, and that of the growing solid phase, $\mu_{i}^{\mathrm{s}, \mathrm{gr}}$. Hence, the superscript gr refers to the growing solid phase. Taking $J_{i}^{+}$equal to $K_{i} a_{i}^{1 \text {,surf }}$, in accordance with chemical reaction rate theory, with $K_{i}$ a kinetic constant (in $\mathrm{m}^{-2} \mathrm{~s}^{-1}$ ) and $a_{i}^{\text {l,surf }}$ the activity of component $i$ in the liquid phase at the surface, the net flux of component $i, R_{i}=J_{i}^{+}-J_{i}^{-}$, is derived to be

$$
R_{i}=K_{i} a_{i}^{1, \text { surf }}\left(1-\exp \left(\frac{\Delta \mu_{i}^{\text {surf }}}{R T^{\text {surf }}}\right)\right)=K_{i}\left(a_{i}^{1, \text { surf }}-a_{i, \mathrm{eq}}^{1, \mathrm{gr}}\right)=K_{i} \sigma_{i}^{\text {surf }}
$$

where $\sigma_{i}^{\text {surf }}=a_{i}^{1 \text {,surf }}-a_{i, \text { eq }}^{\text {l,gr }}$ is the absolute supersaturation for component $i$ at the surface and where we used the familiar expression for the chemical potential difference, being 


$$
\Delta \mu_{i}^{\text {surf }}=R T^{\text {surf }} \ln \left(\frac{a_{i}^{l, \text { surf }}}{a_{i, \text { eq }}^{\text {l,gr }}}\right)
$$

with $a_{i \text {,eq }}^{\text {l,gr }}$ the equilibrium activity of component $i$ in the liquid phase with respect to the growing solid phase of composition $x_{2}^{\mathrm{s}, \mathrm{gr}}\left(=1-x_{1}^{\mathrm{s}, \mathrm{gr}}\right)$, which we are looking for. According to standard thermodynamics, the equilibrium activity of component $i$ in the liquid phase with respect to a growing solid phase of composition $x_{2}^{s, g r}$ is given by

$$
a_{i, \mathrm{eq}}^{1, \mathrm{gr}}=a_{i}^{\mathrm{s}, \mathrm{gr}} C_{i}\left(T^{\mathrm{surf}}\right)
$$

where $C_{i}\left(T^{\text {surf }}\right)$ is equal to

$$
C_{i}\left(T^{\text {surf }}\right) \simeq \exp \left[\frac{\Delta H_{i, 0} \Delta T_{i}^{\text {surf }}}{R T_{i} T^{\text {surf }}}+\frac{\Delta c_{p, i}}{R}\left(\ln \left(\frac{T_{i}}{T^{\text {surf }}}\right)-\frac{\Delta T_{i}^{\text {surf }}}{T^{\text {surf }}}\right)\right]
$$

where $T_{i}$ and $\Delta H_{i, 0}$ are the pure component melting temperature and melting enthalpy at $T_{i}$, respectively, and $\Delta T_{i}^{\text {surf }}=T^{\text {surf }}-$ $T_{i}$. Usually, the term proportional to $\Delta c_{p, i}=c_{p, i}^{\mathrm{l}}-c_{p, i}^{\mathrm{s}}$, i.e., the difference in heat capacity between liquid and solid phase for the pure component $i$, is already quite small within the typical temperature range not too far from the melting temperatures. Further corrections, proportional to $\partial \Delta c_{p, i} / \partial T$, are negligible in most cases. The activity of component $i$ in phase $P$ is a function of the composition of that phase through the relation

$$
a_{i}^{P}=\gamma_{i}^{P} x_{i}^{P}
$$

where $x_{i}^{P}$ is the mole fraction and $\gamma_{i}^{P}$ the activity coefficient of component $i$, which is also composition dependent. The latter quantity is related to the excess mixing Gibbs free energy in phase $\mathrm{P}, G^{P \text {,exc }}$, by

$$
R \ln \left(\gamma_{i}^{P}\right)=\left(\frac{\partial G^{P, \mathrm{exc}}}{\partial N_{i}^{P}}\right)_{P, T, N_{j}(j \neq i)}
$$

where $N_{i}^{P}$ is the amount (in moles) of component $i$ in phase $P$. To describe the excess mixing Gibbs free energy, we have adopted the so-called Redlich-Kister expansion, ${ }^{31}$ which for a binary system reads

$$
G^{P, \mathrm{exc}}=N^{P} x_{1}^{P} x_{2}^{P} \sum_{n=0} g_{n}^{P, \mathrm{exc}}\left(1-2 x_{2}^{P}\right)^{n}
$$

where $N^{P}=\sum_{i} N_{i}^{P}$ is the total number of moles in phase $P$. In general, the expansion coefficients $g_{n}^{\text {exc }}$ are temperature dependent and contain an enthalpy and an entropy part according to $g_{n}^{P \text {,exc }}=h_{n}^{P \text {,exc }}-T s_{n}^{P \text {,exc }}$, with $h_{n}^{P \text {,exc }}$ and $s_{n}^{P \text {,exc }}$ both temperature dependent. However, usually this temperature dependence is weak within the temperature range of interest. ${ }^{32,33}$

From eq 2 and subsequent equations, it follows that the increase of the total amount of solid particles $i, N_{i}^{\mathrm{s}}$, is a function of the growth composition, i.e.,

$$
\frac{\mathrm{d} N_{i}^{\mathrm{s}}}{\mathrm{d} t}=R_{i}\left(x_{2}^{\mathrm{s}, \mathrm{gr}}\right)
$$

For a steady state it holds that $N_{i}^{\mathrm{s}}=x_{i}^{\mathrm{s}, \mathrm{gr}} N^{\mathrm{s}}$, where $N^{\mathrm{s}}=\sum_{i} N_{i}^{\mathrm{s}}$. After substitution of this relation into eq 9 for $i=2$ we obtain

$$
\frac{\mathrm{d} x_{2}^{\mathrm{s}, \mathrm{gr}}}{\mathrm{d} t}=\frac{1}{N^{\mathrm{s}}}\left(R_{2}-x_{2}^{\mathrm{s}, \mathrm{gr}} R\right)
$$

where $R=\mathrm{d} N^{\mathrm{s}} / \mathrm{d} t=\sum_{i} R_{i}$ is the total growth rate. The stable steady-state solution(s) of this differential equation gives us the growth composition(s) we are looking for. They are given by the solutions of

$$
\frac{\mathrm{d} x_{2}^{\mathrm{s}, \mathrm{gr}}}{\mathrm{d} t}=0 \Longrightarrow x_{2}^{\mathrm{s}, \mathrm{gr}}=\frac{R_{2}\left(x_{2}^{\mathrm{s}, \mathrm{gr}}\right)}{R\left(x_{2}^{\mathrm{s}, \mathrm{gr}}\right)}
$$

which in addition have to fulfill the common stability criterion for first order differential equations, being in this case

$$
\frac{\mathrm{d}}{\mathrm{d} x_{2}^{\mathrm{s}, \mathrm{gr}}}\left(R_{2}-x_{2}^{\mathrm{s}, \mathrm{gr}} R\right)<0
$$

at the solution. In most cases, for an undercooled binary system, eq 11 has either one solution, which corresponds to a stable steady state, or three solutions, of which only two fulfill the stability criterion. ${ }^{7}$ In the latter case, two solid phases with different compositions may grow simultaneously if nuclei are available, in which case we speak of kinetic phase separation. Typically, this can occur for eutectic or peritectic systems.

The solutions of eq 11 are equivalent with the solutions of the equation

$$
\frac{x_{1}^{\text {s,gr }}}{x_{2}^{\text {s,gr }}}=\frac{K_{1} \sigma_{1}^{\text {surf }}}{K_{2} \sigma_{2}^{\text {surf }}}=\frac{K_{1}\left(\gamma_{1}^{1, \text { surf }} x_{1}^{1, \text { surf }}-\gamma_{1}^{\text {s,gr }} x_{1}^{\text {s,gr }} C_{1}\left(T^{\text {surf }}\right)\right)}{K_{2}\left(\gamma_{2}^{1, \text { surf }} x_{2}^{1, \text { surf }}-\gamma_{2}^{\text {s,gr }} x_{2}^{\text {s,gr }} C_{2}\left(T^{\text {surf }}\right)\right)}
$$

which is the form in which the LKS model was presented originally. $^{29}$

2.2. Mass Transport Limitation. To find the effective segregation, we have to find a relation between the liquid composition at the surface and that of the bulk liquid phase. To achieve this, we will use basic results from the theory of hydrodynamics for fluids adjacent to a moving and absorbing interphase. For an overview of this theory with a focus on crystal growth we refer to ref 34 .

For a fluid system with convection and not too high viscosity, a condition which is usually well met in crystal growth, a relatively thin, so-called laminar layer is formed in front of the surface, say of width $\Delta_{c}$, in which the flow velocity drops from its bulk value at a distance $\Delta_{c}$ from the surface to zero immediately at the surface, obeying the no-slip condition. Typically, in the case of crystal growth we may have convection caused by stirring or, when no stirring is applied, by free convection, due to temperature and composition gradients in combination with gravity, the so-called buoyancy-driven convection. The laminar layer width, $\Delta_{c}$, depends on the geometry and scale of the system, the kinematic viscosity $v=\eta / \rho$, with $\eta$ the viscosity and $\rho$ the mass density, and on the bulk convective flow velocity, $v_{\mathrm{c}}^{\text {bulk }}$. Generally, $\Delta_{\mathrm{c}}$ is a function of the dimensionless so-called Reynolds number $N_{\operatorname{Re}}=v_{\mathrm{c}}^{\text {bulk }} L / v$ where $L$ a characteristic linear dimension of the flow sytem, e.g., the diameter of the crystallites. Good estimates for $\Delta_{\mathrm{c}}$ have been derived for the most common geometries occurring in crystal growth. In particular, for a rotating disk geometry, appropriate for Czochralski growth, $\Delta_{\mathrm{c}}$ has been derived to be

$$
\Delta_{\mathrm{c}}=4\left(\frac{v}{\omega}\right)^{1 / 2}
$$


where $\omega$ is the angular velocity. For a mixture, on top of convection, diffusion can take place, i.e., the transport of the individual components with respect to the average convective flow velocity. For each component, mass conservation is described by the continuity equation

$$
\frac{\partial c_{i}^{1}}{\partial t}=-\nabla \boldsymbol{J}_{i}=-\nabla \boldsymbol{J}_{i}^{\mathrm{conv}}-\nabla \boldsymbol{J}_{i}^{\mathrm{diff}}
$$

where $c_{i}^{1}$ is the concentration of component $i$ (in moles $\mathrm{m}^{-3}$ ) and $\boldsymbol{J}_{i}$ is the total flux of component $i$, containing a convective part $\boldsymbol{J}_{i}^{\text {conv }}=c_{i}^{\mathrm{l}} \mathbf{v}_{\mathrm{c}}$ and a diffusive part $\boldsymbol{J}_{i}^{\text {diff }}$. For a binary system, $J_{i}^{\text {diff }}$ is given by Fick's first law:

$$
\boldsymbol{J}_{i}^{\mathrm{diff}}=-D \nabla c_{i}^{1}
$$

where $D$ is the diffusion constant. Substitution into eq 15 yields

$$
\frac{\partial c_{i}^{1}}{\partial t}=-\nabla\left(c_{i}^{1} v_{c}\right)+D \nabla^{2} c_{i}^{1}
$$

neglecting the usually small composition dependence of $D$. Hence, the transport of a single component is coupled to the position and time dependent convective flow velocity $\mathbf{v}_{\mathrm{c}}$. Generally, it requires considerable numerical effort to solve the transport equations (Navier Stokes) satisfying the appropriate boundary conditions. However, for mass transport to (from) a surface, it was found that strong gradients occur only in a relatively thin boundary layer, similar as for convection. Owing to the similarity of the diffusive and the convective transport equations, a correlation exists between the width of the mass transport boundary layer, $\Delta_{\mathrm{m}}$, and $\Delta_{\mathrm{c}}$, namely

$$
\Delta_{\mathrm{m}} \simeq\left(\frac{D}{v}\right)^{1 / 3} \Delta_{\mathrm{c}}=\left(\frac{1}{N_{\mathrm{Sc}}}\right)^{1 / 3} \Delta_{\mathrm{c}}
$$

where $N_{\mathrm{Sc}}=v / D$ is the so-called Schmidt number. In general, for liquid phases it holds that $N_{\mathrm{Sc}} \geq 1$, in contrast to vapor phases for which $N_{\mathrm{Sc}}<1$, implying $\Delta_{\mathrm{m}} \leq \Delta_{\mathrm{c}}$ for liquid phases. For many molecular liquids, $\Delta_{\mathrm{m}}$ is even between 1 and 2 orders of magnitude smaller than $\Delta_{\mathrm{c}}$. This conveniently implies that within a distance $\Delta_{\mathrm{m}}$ from the surface we may neglect the first term on the right-hand side of eq 17. Then, assuming a 1-dimensional geometry with a flat surface perpendicular to the $z$-direction, and using the moving boundary coordinate $z^{\prime}=z-v t$, with $v$ the crystal growth velocity and $z^{\prime}=0$ being the position of the suface in the moving frame, eq 17 simplifies to

$$
-v \frac{\mathrm{d} c_{i}^{1}}{\mathrm{~d} z^{\prime}}=D \frac{\mathrm{d}^{2} c_{i}^{1}}{\mathrm{~d} z^{\prime 2}}
$$

The solution of this equation has to fulfill two boundary conditions, namely the condition $c_{i}^{\mathrm{l}}\left(\Delta_{\mathrm{m}}\right)=c_{i}^{\mathrm{l} \text {,bulk }}$ and conservation of mass at the interface. The latter is expressed as

$$
\left.D \frac{\mathrm{d} c_{i}^{1}}{\mathrm{~d} z^{\prime}}\right|_{z^{\prime}=0}+g_{\mathrm{d}} v c_{i}^{1, \text { surf }}=v c_{i}^{\mathrm{s}, \mathrm{gr}}
$$

where the second term on the left-hand side is the advective term due to the motion of the interface into the liquid phase. The factor $g_{\mathrm{d}}$ is a factor that corrects for differences in molar densities. If the solid phase is more dense than the liquid phase, as is usually the case, then the volume of liquid being absorbed within a certain small time interval is larger than the volume of solid-phase being created in that time interval, so that $g_{\mathrm{d}}$ will be larger than one in this case. The "empty" volume being created is filled up by a rigid and almost immediate displacement (with the velocity of sound) of the liquid phase toward the solid phase to recover equality of the pressure everywhere. The solution of eq 19, satisfying the mentioned boundary conditions is given by

$$
\begin{aligned}
& c_{i}^{1}\left(z^{\prime}\right)= \\
& \quad c_{i}^{1, \text { surf }}+\left(\frac{c_{i}^{1, \text { bulk }}-c_{i}^{1, \text { surf }}}{1-\exp \left(-v \Delta_{\mathrm{m}} / D\right)}\right)\left(1-\exp \left(-v z^{\prime} / D\right)\right)
\end{aligned}
$$

where $c_{i}^{1 \text {,surf }}=c_{i}^{1}(0)$ follows by substitution of eq 21 into eq 20 . For the mole fraction $x_{i}^{1, \text { surf }}=c_{i}^{1 \text {,surf }} / c^{1 \text {,surf }}$, with $c^{1 \text {,surf }}=\sum_{i} c_{i}^{1 \text {,surf }}$ the total concentration at the surface, this gives

$$
x_{i}^{1, \text { surf }}=\frac{g_{1, \text { surf }}^{\text {s,gr }} x_{i}^{\text {s,gr }}+\left(g_{1, \text { surf }}^{1, \text { bulk }} x_{i}^{1, \text { bulk }}-g_{1, \text { surf }}^{\text {s,gr }} x_{i}^{\text {s,gr }}\right) \exp \left(q_{\mathrm{m}} \tilde{\sigma}^{\text {surf }}\right)}{\left(g_{\mathrm{d}}+\left(1-g_{\mathrm{d}}\right) \exp \left(q_{\mathrm{m}} \tilde{\sigma}^{\text {surf }}\right)\right)}
$$

where we defined the density ratios $g_{1, \text { surf }}^{\mathrm{s}, \mathrm{gr}} \equiv c^{\mathrm{s}, \mathrm{gr}} / c^{\mathrm{l} \text {,surf }}$ and $g_{1, \text { surf }}^{\mathrm{l} \text {, } u l k}$ $\equiv c^{\mathrm{l} \text {, bulk }} / c^{\mathrm{l} \text {,surf }}$ with $c^{\mathrm{l} \text {,bulk }}=\sum_{i} c_{i}^{1 \text {,bulk }}$. The parameter $q_{\mathrm{m}}$ in eq 22 is the crucial parameter for mass transport limitation. It is defined as

$$
q_{\mathrm{m}}=\frac{\hat{v} \Delta_{\mathrm{m}}}{D_{\mathrm{m}}}
$$

where $\hat{v}$ (in $\mathrm{m} / \mathrm{s}$ ) is an average velocity constant given by

$$
\hat{v}=\frac{1}{2} \sum_{i=1}^{2} K_{i} V_{i}^{\mathrm{s}, \mathrm{gr}}
$$

with $V_{i}^{\text {s,gr }}$ the volume per particle of component $i$ in the growing solid phase. The quantity $\tilde{\sigma}^{\text {surf }}$ in eq 22 is a weighted supersaturation defined as

$$
\tilde{\sigma}^{\text {surf }}=\sum_{i=1}^{2} \frac{K_{i} V_{i}^{\mathrm{s}, \mathrm{gr}}}{\hat{v}} \sigma_{i}^{\mathrm{surf}}=\sum_{i=1}^{2} \frac{K_{i} V_{i}^{\mathrm{s}, \mathrm{gr}}}{\hat{v}}\left(\gamma_{i}^{1, \mathrm{surf}} x_{i}^{1, \mathrm{surf}}-\gamma_{i, \mathrm{eq}}^{1, \mathrm{gr}} x_{i, \mathrm{eq}}^{1, \mathrm{gr}}\right)
$$

According to the LKS model, the crystal growth velocity is equal to $v=\hat{v} \tilde{\sigma}^{\text {surf }}$, which implies the relation

$$
q_{\mathrm{m}} \tilde{\sigma}^{\text {surf }}=\frac{v \Delta_{\mathrm{m}}}{D}
$$

If we sum eq 22 for $i=1$ and 2 and use $x_{1}^{P}+x_{2}^{P}=1$ for each phase $P=(1$, bulk), (1,surf) and (s,gr), we obtain a relation for $g_{\mathrm{d}}$, which is

$$
g_{\mathrm{d}}=\frac{g_{1, \text { surf }}^{\text {s,gr }}+\left(g_{1, \text { surf }}^{1, \text { bulk }}-g_{1, \text { surf }}^{\text {s,gr }}-1\right) \exp \left(q_{\mathrm{m}} \tilde{\sigma}^{\text {surf }}\right)}{1-\exp \left(q_{\mathrm{m}} \tilde{\sigma}^{\text {surf }}\right)}
$$

This equation gives $g_{\mathrm{d}}=1$ if all densities are equal, as it should be. The total molar density in a phase $P$ is equal to $c^{P}=$ $1 / V_{\text {mix }}^{P}$, where $V_{\text {mix }}^{P}$ is the average volume per mole in the mixed phase, which can be written as:

$$
V_{\text {mix }}^{P}=\sum_{i} x_{i} V_{i}^{P}+V^{P, \operatorname{exc}}\left(x_{1}, x_{2}\right)
$$

where $V_{i}^{P}$ is the partial molar volume of the pure component $i$ 
in phase $P$ and $V^{P, \text { exc }}$ the excess volume. In many cases $V^{P, \text { exc }}$ is quite small and can be neglected. Then only the pure component specific volumes are required, and the density ratio between phase $P$ and $P^{\prime}$ becomes

$$
g_{P^{\prime}}^{P}=\frac{c^{P}}{c^{P^{\prime}}}=\frac{x_{1}^{P^{\prime}} V_{1}^{P^{\prime}}+x_{2}^{P^{\prime}} V_{2}^{P^{\prime}}}{x_{1}^{P} V_{1}^{P}+x_{2}^{P} V_{2}^{P}}
$$

It can be shown that in the limit that the composition at the surface takes the equilibrium concentration, i.e., $x_{i}^{1, \text { surf }}=x_{i \text {,eq }}^{1}$, and assuming equal densities $g_{1, \text { surf }}^{\text {s,gr }}=g_{1 \text {, surf }}^{\text {s,bulk }}=g_{\mathrm{d}}=1$, eq 22 becomes equivalent to the BPS equation ${ }^{14}$ mentioned in the Introduction.

An estimate of the kinetic constant $\hat{v}$ could be obtained from measured growth velocities. For a known (measured) growth velocity $v$, the quantity $q_{\mathrm{m}} \tilde{\sigma}^{\text {surf }}$ in eq 22 can be evaluated using eq 26 for a known diffusion constant and an estimate for $\Delta_{m}$ from eqs 14 and 18. Then, for given liquid bulk composition and temperature, eq 22, inserting eqs 27 and 29 for the density factors, can be solved (numerically) simultaneously with eq 11 (or eq 13), giving the growth composition of the solid phase and the composition of the liquid at the surface. At the same time it provides the individual supersaturations $\tilde{\sigma}_{i}^{\text {surf }}$. Then, assuming $K_{1} \simeq K_{2}$, as an often used reasonable approximation for isomorphous components yielding solid solutions, $\hat{v}=v / \widetilde{\sigma}^{\text {surf }}$ can be determined using eqs 24 and 25 .

Once $\hat{v}$ is known, $q_{\mathrm{m}}$ can be determined using eq 23 . Then, the set of coupled equations, consisting of eq 22 for $i=2$ (or equivalently $i=1$ ), including eqs 27 and 29, eq 11 (or eq 13), and the two stoichiometric relations $\sum_{1}^{2} x_{i}^{\mathrm{s}, \mathrm{gr}}=1$ and $\sum_{1}^{2} x_{i}^{\mathrm{l} \text {,surf }}=$ 1 can be solved for any bulk liquid composition and temperature, giving $x_{i}^{\mathrm{s}, \mathrm{gr}}$ and $x_{i}^{\text {, surf }}$ for $i=1,2$. This enables the construction of EKPDs for isothermal conditions, i.e., neglecting heat transport limitation. The nonisothermal extension of the LEKS model is described in the next section.

2.3. Heat Transport Limitation. Under normal circumstances encountered in crystal growth, energy conservation within the liquid phase in good approximation reduces to a rather simple transport equation, ${ }^{34}$ which is similar to eq 17 for mass transport, namely:

$$
c^{1} c_{\mathrm{p}}^{1} \frac{\partial T}{\partial t}=-c^{1} c_{\mathrm{p}}^{1} \nabla\left(\mathbf{v}_{\mathrm{c}} T\right)-\nabla \mathbf{J}_{q}^{1}=-c^{1} c_{\mathrm{p}}^{1} \nabla\left(\mathbf{v}_{\mathrm{c}} T\right)+k^{1} \nabla^{2} T
$$

where $\mathbf{J}_{q}^{1}=-k^{1} \nabla T$ is the heat flux according to Fourier's law with $k^{1}$ the thermal conductivity in the liquid phase. We note that in eq 30 we have neglected the contribution from radiation, which is usually small and can effectively be included in the "normal" heat conduction, from which it is hardly distuiguishable. In contrast to mass transport, heat can also be transported via the solid phase. So, for the solid phase we have an equation similar to eq 30 , but without the convective term, since $v_{\mathrm{c}}=0$ within the solid phase.

Now we will discuss the heat transport in the liquid phase. In analogy with the mass transport problem, for a system with convection, strong temperature gradients occur only within a relatively thin, thermal boundary layer of width $\Delta_{\mathrm{T}}^{\mathrm{l}}$, which is related to $\Delta_{\mathrm{c}}$ (and $\Delta_{\mathrm{m}}$ ) by

$$
\Delta_{\mathrm{T}}^{1} \simeq\left(\frac{D_{\mathrm{T}}^{1}}{v}\right)^{1 / 3}=\left(\frac{1}{N_{\mathrm{Pr}}}\right)^{1 / 3} \Delta_{\mathrm{c}}=\left(\frac{D_{\mathrm{T}}^{1}}{D}\right)^{1 / 3} \Delta_{\mathrm{m}}
$$

where $D_{\mathrm{T}}^{1}=k^{1} /\left(c^{1} c_{\mathrm{p}}^{1}\right)$ is the thermal diffusivity in the liquid phase and $N_{\mathrm{Pr}}=v / D_{\mathrm{T}}^{1}$ is the so-called Prandtl number. Similar to the mass transport problem, we may solve eq 30 omitting the convective term, but respecting the boundary condition $T\left(z^{\prime}\right.$ $\left.=\Delta_{\mathrm{T}}^{\mathrm{l}}\right)=T^{\text {, bulk }}$ with the $z^{\prime}$ coordinate in the moving frame, as already used above, and $T^{\text {,bulk }}$ the bulk liquid temperature, which can be taken as the applied temperature. Then, in a 1-d geometry, neglecting the temperature dependence of $k^{1}$, eq 30 becomes

$$
-v \frac{\mathrm{d} T}{\mathrm{~d} z^{\prime}}=D_{\mathrm{T}}^{1} \frac{\mathrm{d}^{2} T}{\mathrm{~d} z^{\prime 2}} \quad z^{\prime}>0
$$

The solution of this equation satisfying also the second boundary condition $T(0)=T^{\text {surf }}$ is given by

$$
\begin{aligned}
& T\left(z^{\prime}\right)= \\
& \frac{T^{\text {, bulk }}-\exp \left(-q_{\mathrm{T}}^{1} \tilde{\sigma}^{\text {surf }}\right) T^{\text {surf }}-\left(T^{\text {, bulk }}-T^{\text {surf }}\right) \exp \left(-v z^{\prime} / D_{\mathrm{T}}^{1}\right)}{1-\exp \left(-q_{\mathrm{T}}^{1} \tilde{\sigma}^{\text {surf }}\right)}
\end{aligned}
$$

for $z^{\prime}>0$ where $q_{\mathrm{T}}^{1}$ is the crucial parameter for heat transport limitation defined as

$$
q_{\mathrm{T}}^{1}=\frac{\hat{v} \Delta_{\mathrm{T}}^{1}}{D_{\mathrm{T}}^{1}}
$$

implying $q_{\mathrm{T}}^{1} \tilde{\sigma}^{\text {surf }}=v \Delta_{\mathrm{T}}^{1} / D_{\mathrm{T}}^{1}$.

For heat transport in the solid phase, eq 30 directly simplifies to eq 32 , but with the superscript 1 replaced by s. The solution with boundary condition $T\left(-\Delta_{\mathrm{T}}^{\mathrm{s}}\right)=T^{\text {s, bulk }}$, with $T^{\text {s, bulk }}$ the temperature inside the solid phase at a distance $\Delta_{\mathrm{T}}^{\mathrm{s}}$ from the interface, is similar to eq 33 , but with $-q_{T}^{l} \tilde{\sigma}^{\text {surf }}$ replaced by $q_{T}^{s} \tilde{\sigma}^{\text {surf }}$ where

$$
q_{\mathrm{T}}^{\mathrm{s}}=\frac{\hat{v} \Delta_{\mathrm{T}}^{\mathrm{s}}}{D_{\mathrm{T}}^{\mathrm{s}}}
$$

implying $q_{\mathrm{T}}^{\mathrm{s}} \tilde{\sigma}^{\text {surf }}=v \Delta_{\mathrm{T}}^{\mathrm{s}} / D_{\mathrm{T}}^{\mathrm{s}}$. The surface temperature $T^{\text {surf }}$ follows from the heat conservation boundary condition

$$
c^{\mathrm{s}, \mathrm{gr}} v \Delta H^{\mathrm{s}, \mathrm{gr}}=J_{q, 0}^{\mathrm{l}}+J_{q, 0}^{\mathrm{s}}=-\left.k^{1} \frac{\mathrm{d} T}{\mathrm{~d} z}\right|_{z^{\prime} \downarrow 0}+\left.k^{\mathrm{s}} \frac{\mathrm{d} T}{\mathrm{~d} z}\right|_{z^{\prime} \uparrow 0}
$$

where $J_{q, 0}^{\mathrm{l}}$ and $J_{q, 0}^{\mathrm{s}}$ are the heat fluxes into the liquid and the solid phase respectively and where $\Delta H^{\mathrm{s}, \mathrm{gr}}=\Delta H^{\mathrm{s}, \mathrm{gr}}\left(x_{2}^{\mathrm{s}, \mathrm{gr}}, T^{\mathrm{surf}}\right)$ is the molar, composition, and temperature-dependent melting enthalpy of the growing solid phase given by

$$
\Delta H^{\mathrm{s}, \mathrm{gr}}=\sum_{i} x_{i}^{\mathrm{s}, \mathrm{gr}}\left(\Delta H_{i, 0}+\Delta H_{i}^{\mathrm{exc}}+\int_{T_{i}}^{T^{\mathrm{surf}}} \Delta c_{p, i} \mathrm{~d} T^{\prime}\right)
$$

where $\Delta H_{i}^{\text {exc }}=H_{i}^{\mathrm{l} \text { exc }}-H_{i}^{\mathrm{s} \text { exc }}$ is the difference in the partial excess enthalpies of component $i$ in the liquid and the solid phase. To determine $J_{q, 0}^{\mathrm{s}}=k^{\mathrm{s}} \mathrm{d} T /\left.\mathrm{d} z\right|_{z^{\uparrow} \uparrow 0}$, we have to assume values for $\Delta_{\mathrm{T}}^{\mathrm{s}}$ and $T^{\mathrm{s}}$,bulk. For this purpose we consider two cases regarding to the situation of the crystallites. Either the crystallite is floating in the fluid phase or it is attached to the wall, corresponding to homogeneous and heteregeneous nucleation, respectively. In the former case, since there is no cooling source inside the crystallite, the temperature will be constant throughout the crystallite and will be equal to the surface 


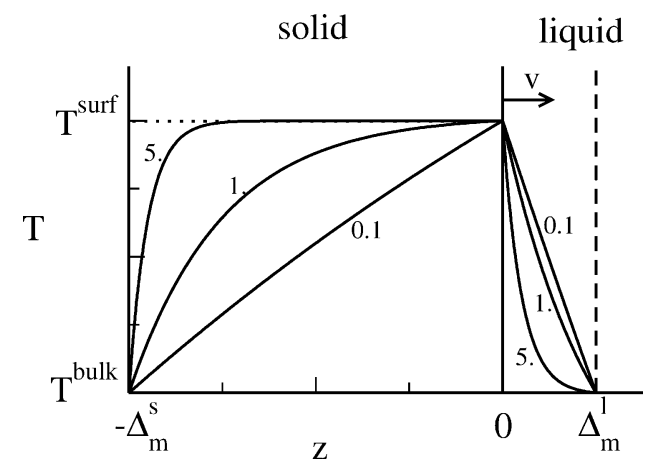

Figure 2. Schematic illustration of heat transport limitation. Temperature profiles for $q_{\mathrm{T}}^{1}=0.1,1.0$, and 5.0 are shown in a situation where a crystallite is sticking to the wall at a distance $\Delta_{\mathrm{T}}^{\mathrm{s}}=4 \Delta_{\mathrm{T}}^{1}$ from the solification front. We assumed that $D_{\mathrm{T}}^{\mathrm{s}}=D_{\mathrm{T}}^{1}$, implying $q_{\mathrm{T}}^{\mathrm{s}}=4 q_{\mathrm{T}}^{1}$.

temperature, implying $J_{q, 0}^{\mathrm{s}}=0$. Then, substitution of eq 33 into eq 36 leads to

$$
T^{\text {surf }}=T^{\text {l,bulk }}+\left(1-\exp \left(-q_{\mathrm{T}}^{1} \tilde{\sigma}^{\text {surf }}\right)\right) \frac{c^{\mathrm{s}} \Delta H^{\mathrm{s}, \mathrm{gr}}}{c^{1} c_{\mathrm{p}}^{1}}
$$

In the other case, if the crystallite is sticking to the wall, $T^{\mathrm{s} \text {,bulk }}$ should be taken equal to the wall temperature, $T^{\text {wall }}$, and correspondingly $\Delta_{\mathrm{T}}^{\mathrm{s}}$ should be equal to the distance between the wall and the solidification front which increases during the growth. Assuming that we may still use the steady-state solution but with $\Delta_{\mathrm{T}}^{\mathrm{s}}$ slowly varying in time, and taking $T^{\mathrm{s} \text {, bulk }}=T^{\text {wall }}=$ $T^{\text {l,bulk }}$ as the applied temperature, eq 36 leads to

$$
\begin{aligned}
T^{\text {surf }}= & T^{\text {, bulk }}+ \\
& \frac{c^{\mathrm{s}} \Delta H^{\text {s,gr }}\left(\exp \left(q_{\mathrm{T}}^{\mathrm{s}} \tilde{\sigma}^{\text {surf }}\right)-1\right)\left(1-\exp \left(-q_{\mathrm{T}}^{1} \tilde{\sigma}^{\text {surf }}\right)\right)}{c^{1} c_{\mathrm{p}}^{1} \exp \left(q_{\mathrm{T}}^{s} \tilde{\sigma}^{\text {surf }}-1\right)+c^{\mathrm{s}} c_{\mathrm{p}}^{\mathrm{s}}\left(1-\exp \left(-q_{\mathrm{T}}^{1} \tilde{\sigma}^{\text {surf }}\right)\right)}
\end{aligned}
$$

Typical temperature profiles according to eq 39 for different values of the parameter $q_{\mathrm{T}}^{\mathrm{l}}$ with $q_{\mathrm{T}}^{\mathrm{s}}=4 q_{\mathrm{T}}^{\mathrm{l}}$ and $\Delta_{\mathrm{T}}^{\mathrm{s}}=4 \Delta_{\mathrm{T}}^{\mathrm{l}}$ are shown in Figure 2. As shown, the motion of the interface introduces an asymmetry in the profiles which is convex at the liquid side and concave at the solid side, implying a larger gradient, i.e., larger heat flux, at the liquid side. This difference in the fluxes further increases when $\Delta_{\mathrm{T}}^{\mathrm{s}}>\Delta_{\mathrm{T}}^{\mathrm{l}}$. Eventually, after some time during the growth $\Delta_{\mathrm{T}}^{\mathrm{s}} \gg \Delta_{\mathrm{T}}^{1}$ and $J_{q, 0}^{\mathrm{s}}$ becomes negligible compared to $J_{q, 0}^{1}$. Thus, also in this case the dominant heat flux will be that into the liquid phase, implying that eq 38 should be a good approximation for $T^{\text {surf }}$ for both cases regarding the situation of the crystallites. Therefore, for the calculations presented in the next section we used eq 38 .

Summarizing, including heat transport limitation, the LEKS model is completed with one more equation, eq 38 including eq 37 , and one extra unknown, $T^{\text {surf }}$, to be solved together with the equations mentioned at the end of section 2.2.

\section{Results}

To explore the parameter space of the LEKS model, we have made a limited database covering different classes of materials, including a selection of metals, semiconductors, and mediumsized organic molecular systems. The latter group was restricted to $n$-alkanes $(n \leq 20)$, simple aromatic systems, and fat systems. Many mixtures of such systems show good miscibility in the solid phase. ${ }^{32,36-38}$ Table 1 gives a list of lower and upper bounds for the values of the relevant parameters for each group of materials resulting from our database. For the calculations
TABLE 1: Rough Bounds for the Most Important Parameters of the LEKS Model Resulting from a Database for Three Classes of Materials ${ }^{a}$

\begin{tabular}{llll}
\hline & \multicolumn{1}{c}{ metals } & semiconductors & molecular systems \\
\hline$\Delta H_{\mathrm{m}} / R T_{\mathrm{m}}$ & $0.6-1.7$ & $2.5-4.3$ & $3.3-72.0$ \\
$c_{\mathrm{P}}^{1} / R$ & $2.3-4.7$ & $2.6-4.6$ & $12.0-250.0$ \\
$\Delta H_{\mathrm{m}} / c_{\mathrm{p}}^{1}(\mathrm{~K})$ & $50-800$ & $800 .-2200$. & $28 .-130$. \\
$D_{\mathrm{m}}\left(10^{-4} \mathrm{~cm} / \mathrm{s}^{2}\right)$ & $4.5-55.0$ & $4.6-8.4$ & $0.003-1.8$ \\
$D_{\mathrm{T}}\left(\mathrm{cm} / \mathrm{s}^{2}\right)$ & $0.18-1.55$ & $0.6-2.1$ & $0.00014-0.0012$ \\
$\Delta_{\mathrm{c}}(\mathrm{cm})$ & $0.034-0.090$ & $0.09-0.23$ & $0.13-3.30$ \\
$\Delta_{\mathrm{m}}(\mathrm{cm})$ & $0.058-0.16$ & $0.07-0.13$ & $0.018-0.075$ \\
$\Delta_{\mathrm{T}}(\mathrm{cm})$ & $0.37-1.10$ & $0.77-1.8$ & $0.06-0.21$ \\
$v(\mathrm{~cm} / \mathrm{s})$ & 0.005 & 0.0005 & 0.00005 \\
$q_{\mathrm{m}} \sigma\left(10^{-1}\right)$ & $1.1-8.0$ & $0.6-1.0$ & $0.16-32.0$ \\
$q_{\mathrm{T}}^{1} \sigma\left(10^{-1}\right)$ & $0.03-0.15$ & $0.0034-0.0076$ & $0.055-1.20$
\end{tabular}

${ }^{a}$ The data for molecular systems refer to medium size molecules ranging from benzene to fat molecules. The width of the convective boundary is based on eq 14, taking a stirring speed equal to $\omega=15$ $\mathrm{rpm}$. The values of $q_{m} \sigma^{\text {surf }}$ and $q_{T}^{l} \sigma^{\text {surf }}$ are based on the given crystal growth velocities $v$, representing the order of magnitude of typical experimentally observed velocities.

presented here, Table 1 was used as a rough guide in the parameter space. We present effective kinetic phase diagrams (EKPDs) for systems belonging to each of the three classes of systems. In all cases we assumed ideal miscibility in the liquid phase, i.e., $G^{\text {,exc }}=0$. We note that what matters here mostly is the difference in free energy between liquid and solid phase, so that a possible not-zero excess energy for the liquid phase can be put into the excess energy for the solid phase. Furthermore, we have neglected small contributions, ignoring the temperature dependencies of the diffusivities and viscosities, assuming equal liquid and solid heat capacities, i.e., $\Delta c_{\mathrm{p}, \mathrm{i}}=0$, and equal molar densities, i.e., $g_{1, \text { surf }}^{\mathrm{s}, \mathrm{gr}}=g_{1, \text { surf }}^{\mathrm{s} \text {,bulk }}=1$. We also assumed that $K_{1}=K_{2}$, implying $\tilde{\sigma}=\sigma$.

With the given typical crystal growth velocities in Table 1, the quantity $q_{\mathrm{m}} \sigma$ can be estimated, and from this $q_{\mathrm{m}}$ can be determined as explained at the end of section 2. It is important to note that, due to the correlation between the boundary layers for mass and heat transport, the ratio between $q_{\mathrm{m}}$ and $q_{\mathrm{T}}^{1}$ is a material property. So even with the lack of an accurate value for $q_{\mathrm{m}}$, due to the uncertainty in $\hat{v}$ and/or $\Delta_{\mathrm{m}}$, once we have assumed a value for it, within a reasonable range, the value of $q_{\mathrm{T}}^{1}$ is fixed due to eq 31 by quantities that are usually wellknown or easily accessible. This allows for a sensible, comparative study of the relative contributions of mass and heat transport limitation to the effective segregation for different materials. More specifically, from our database we find that the ratio $q_{\mathrm{T}}^{1} / q_{\mathrm{m}}$ lies within the interval [0.008-0.04] for most metals, within [0.005-0.01] for semiconductors and within [0.05-0.7] for molecular systems. Thus, for molecular systems this ratio is about an order of magnitude larger than for both other systems. In the figures presented in this section we use the dimensionless bulk liquid temperature $\theta=T / T_{2}$ and, accordingly, the relative bulk undercooling $\Delta \theta$, being defined as:

$$
\Delta \theta=\frac{\Delta T}{T_{2}}=\frac{T_{\mathrm{eq}}-T^{\mathrm{l}, \mathrm{bulk}}}{T_{2}}
$$

where $T_{2}$ is the melting temperature of the component with the highest melting temperature, which is component 2 in all cases, and where $T_{\text {eq }}$ is the equilibrium temperature for the given bulk liquid mixture.

Figure 3 shows the EKPDs according to the LEKS model for a typical medium size molecular system, with thermodynamic properties given in the figure caption, for three relative 

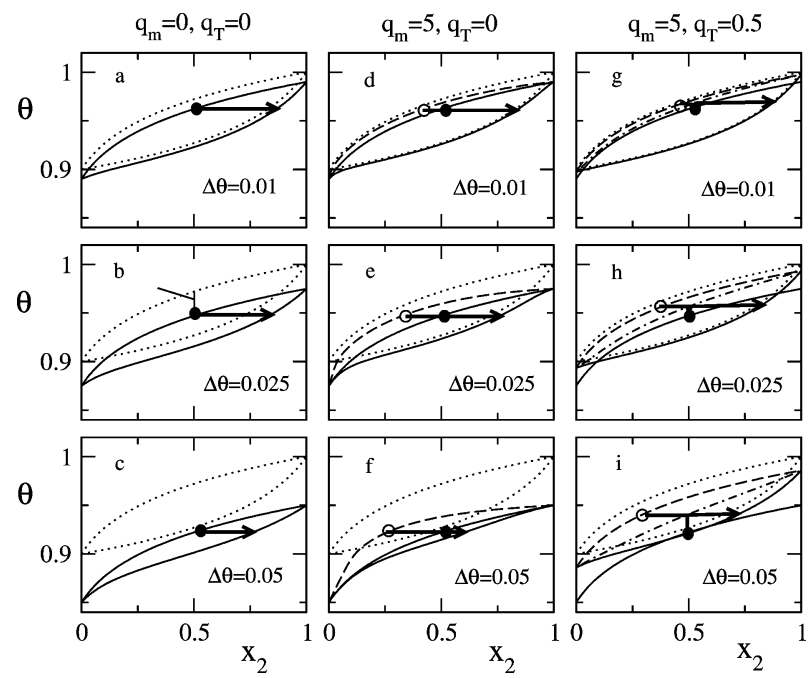

Figure 3. Effective kinetic phase diagrams (full lines) for a binary model system of medium sized molecules with ideal miscibility, i.e., $G^{\mathrm{s}, \mathrm{exc}}=0$. The used pure component data are $\Delta H_{1,0} / R T_{2}=18, \Delta H_{2,0} /$ $R T_{2}=20, c_{\mathrm{p}, 1}^{1} / R=c_{\mathrm{p}, 2}^{1} / R=20.0$, and $T_{1} / T_{2}=0.9$. The vertical axis is in units of the relative temperature $\theta=T / T_{2}$. Results are shown for different relative undercoolings $\Delta \theta$ for the case without transport limitation (graphs a, b, and c), the case with only mass transport limitation (graphs d, e, and f), and the case including both mass and heat transport limitation (graphs $\mathrm{g}, \mathrm{h}$, and i), as also indicated by the values of $q_{\mathrm{m}}$ and $\mathrm{q}_{\mathrm{T}}^{1}$ at the top of each column of graphs. The surface kinetic liquidus (dashed line) gives the composition of the liquid phase at the surface. The dashed-dotted line is a help line giving the temperature at the surface. The dotted lines represent the equilibrium phase diagram.

bulk undercoolings $\Delta \theta$. To illustrate the magnitude of the various contributions to the effective segregation, results are shown of calculations (i) without including transport limitations (i.e., $q_{\mathrm{m}}=q_{\mathrm{T}}^{\mathrm{l}}=0$ ), (ii) including only mass transport limitation (i.e., $q_{\mathrm{T}}^{1}=0$ ), and (iii) including both mass and heat transport limitation. In all cases the kinetic liquidus is simply constructed by a downward shift of the equilibrium liquidus over a distance $\Delta \theta$. To demonstrate how to read these graphs, we have indicated the effective segregation for one particular point on the kinetic liquidus at a mole fraction equal to 0.5 , shown as a closed dot. A measure for the effective segregation is given by the horizontal distance between this dot on the kinetic liquidus and the arrowhead ending at the growth composition on the kinetic solidus. The corresponding steady-state liquid composition at the surface in the case of mass transport limitation (i.e., $q_{\mathrm{m}} \neq 0$ ) is indicated by the open dot on the surface kinetic liquidus (dashed lines). For the case with both mass and heat transport limitation (i.e., $q_{\mathrm{T}}^{1} \neq 0$ ), an additional help line is required giving the temperature at the interface (dashed-dotted line). In this case, for a given point on the kinetic liquidus, the growth compostion is found by starting from this point, first moving vertically upward until this help line is crossed and then moving horizontally toward the kinetic solidus. The corresponding surface liquid composition is found by the intersection of that horizontal line with the surface kinetic liquidus (dashed line).

The results in Figure 3 can be summarized as follows. The segregation decreases with increasing bulk undercooling. Mass transport limitation reduces the effective segregation further. Heat transport limitation reduces the effective undercooling at the interface and therefore it tempers the effects of interfacial undercooling and mass transport, thus enhancing the effective segregation (getting closer to the equilibrium segregation).

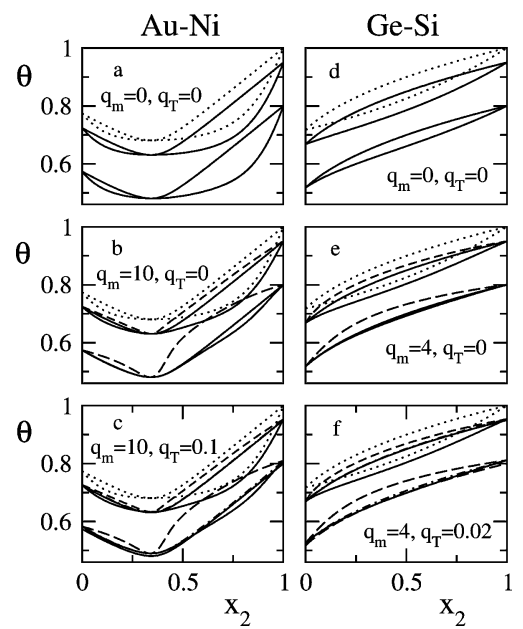

Figure 4. Calculated EKPDs (full lines) for binary mixtures of $\mathrm{Au} / \mathrm{Ni}$ (left column of graphs) and $\mathrm{Ge} / \mathrm{Si}$ (right column of graph) for two relative undercoolings of $\Delta \theta=0.05$ and $\Delta \theta=0.2$, with and without including mass and heat transport limitation, as indicated by the shown values of $q_{\mathrm{m}}$ and $q_{\mathrm{T}}^{1}$. The dotted lines give the equilibrium phase diagrams.

TABLE 2: Pure Component Thermodynamic Properties and Dimensionless Excess Energy Parameter $g^{\mathrm{s}, \operatorname{exc}_{0} / R T_{\text {av }}}\left(T_{\text {av }}\right.$ $\left.=\left(T_{1}+T_{2}\right) / 2\right)$ of the Components of Figures 3 and 4

\begin{tabular}{lcccccc}
\hline & $\mathrm{Au}$ & $\mathrm{Ni}$ & $\mathrm{Ge}$ & \multicolumn{1}{c}{$\mathrm{Si}$} & $\mathrm{EEE}$ & $\mathrm{SSS}$ \\
\hline $\mathrm{T}_{\mathrm{m}}$ & 1337 & 1728 & 1211 & 1687 & 315.35 & 345.65 \\
$\Delta H_{\mathrm{m}} / R T_{\mathrm{m}}$ & 1.12 & 1.20 & 3.16 & 3.58 & 56.45 & 67.57 \\
$c_{\mathrm{P}}^{1} / R$ & 3.06 & 3.14 & 3.82 & 3.27 & 216.5 & 216.5 \\
$g_{0}^{\text {s,exc }} / R T_{\text {av }}$ & \multicolumn{2}{c}{0.887} & \multicolumn{2}{c}{0.0} & \multicolumn{2}{c}{0.0}
\end{tabular}

To investigate the tendencies for different classes of materials, we have calculated EKPDs for three binary mixtures from each class included in Table 1, using realistic values of the parameters. The mixtures we have chosen are the metallic gold/ nickel $(\mathrm{Au} / \mathrm{Ni})$ mixture, the semiconductor mixture of silicon/ germanium $(\mathrm{Ge} / \mathrm{Si})$ and a molecular mixture of the two fats trielaidine and tri-stearine, denoted as EEE and SSS respectively according to a conventional nomenclature, where the three letters specify the three fatty acids that are esterified with the glycerol forming a triglyceride. E stands for elaidic and $\mathrm{S}$ for stearic, both having a chain length of $18 \mathrm{C}$ atoms. The relevant pure component thermodynamic properties for all components in the three mixtures are given in Table 2 . The $\mathrm{Au} / \mathrm{Ni}$ system exhibits nonideal mixing in the solid phase, yielding an azeotropic equilibrium phase diagram, ${ }^{35}$ which could be approximated well by assuming a single nonzero excess parameter $g_{0}^{\mathrm{s}, \mathrm{exc}} / R T_{\mathrm{av}}$ for the solid phase. The mixing behavior of both other systems is taken to be ideal, in accordance with refs 13 and 38 .

The calculated EKPDs for two different bulk undercoolings, with and without taking into account mass and heat transport limitation, are shown in Figure 4 for the $\mathrm{Au} / \mathrm{Ni}$ and $\mathrm{Ge} / \mathrm{Si}$ mixture and in Figure 5 for the fat mixture. For the metallic mixture the effect of the interfacial undercooling, which for the case without heat transport limitation (i.e., $q_{\mathrm{T}}^{1}=0$ ) is just equal to the bulk undercooling, on the effective segregation is relatively small. Even at a bulk undercooling of $\Delta \theta=0.15$, the EKPD looks almost the same as the equilibrium phase diagram (see Figure 4a), but shifted to lower temperatures. This is typical for systems with small melting entropies $\Delta S_{\mathrm{m}}=\Delta H_{\mathrm{m}} /$ $T_{\mathrm{m}}$. Furthermore, the effect of mass transport limitation on the segregation is much stronger than that of heat transport limitation, as can be concluded by comparing Figure $4 \mathrm{~b}$ and 4c. Due to the relatively fast heat transport for metals the 

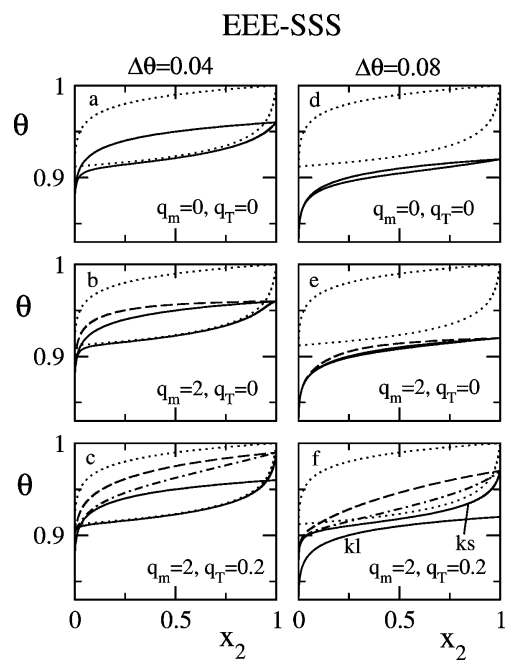

Figure 5. Calculated EKPDs (full lines) and equilibrium phase diagrams (dotted lines) for the fat mixture EEE-SSS for relative undercoolings $\Delta \theta=0.04$ (left column of graphs) and $\Delta \theta=0.08$ (right column of graph). In graphs $\mathrm{f}$ we have indicated the kinetic liquidus and solidus as $\mathrm{kl}$ and $\mathrm{ks}$ respectively.

temperature at the surface remains almost equal to that of the bulk liquid phase during growth. This holds also for the semiconductor mixture. However, for the Ge/Si mixture the effect of the interfacial undercooling on the effective segregation is stronger than for the $\mathrm{Au} / \mathrm{Ni}$ system, as can be seen by comparing Figure $4 \mathrm{a}$ and $4 \mathrm{~d}$. For the Ge/Si system, the segregation is reduced significantly for $\Delta \theta=0.15$. It is further reduced when mass transport limitation is included (Figure $4 \mathrm{e}$ ). Taking into account also heat transport limitation hardly changes the effective segregation further anymore (Figure 4f). For the fat system, the situation is again different. The effect of the interfacial undercooling on the effective segregation is quite strong (Figure 5a and 5d). Actually, one could say that it overrules the effect of mass transport limitation, as becomes clear by comparing Figure $5 \mathrm{~b}$ and $5 \mathrm{e}$ with Figure $5 \mathrm{a}$ and $5 \mathrm{~d}$, respectively. However, adding also heat transport limitation leads again to stronger effective segregation, as can be verified by applying the procedure as explained in the discussion of Figure 3. Starting at some point somewhere on the kinetic liquidus will end up in a point on the kinetic solidus corresponding to a significantly higher concentration of component 2 then that of the starting point.

These differences between the different types of materials, as shown in Figures 4 and 5 are striking and characteristic. It shows that for solid solution growth in molecular mixtures heat transport limitation is much stronger than for atomic mixtures where mass transport limitation is dominant. This is further illustrated in Figure 6, where we show the effective segregation coefficient $k_{\mathrm{eff}, 1}=x_{1}^{\mathrm{s}, \mathrm{gr}} / x_{1}^{1}$ as a function of the bulk undercooling for the $\mathrm{Au} / \mathrm{Ni}$ system (Figure 6a) and the EEE/SSS system (Figure 6b). The compositions of the two systems in mole fractions were chosen to be $0.4 / 0.6$ and $0.8 / 0.2$, respectively. These compositions yield a strong equilibrium segregation, i.e., the segregation for $\Delta \theta \rightarrow 0$, in both cases. For the fat system (Figure 6b), the dotted line, which gives the result without transport limitations, lies between those with only mass transport limitation (dashed lines) and those including both mass and heat heat transport limitation (full line), confirming the above statement that for molecular mixtures heat transport limitation can significantly enlarge the effective segregation, tempering the effects of the interfacial undercooling and mass transport limitation, in contrast to the semiconductor and metallic systems.

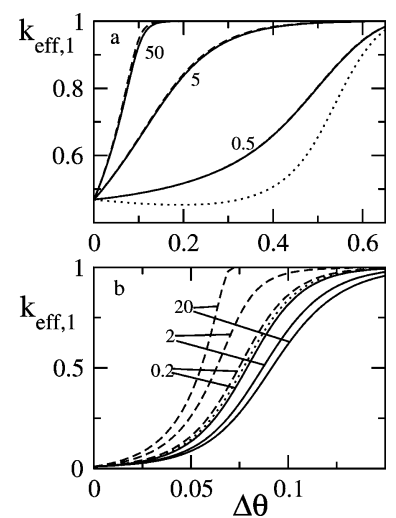

Figure 6. Effective segregration coefficient $k_{1, \text { eff }}=x_{1}^{\mathrm{s}, \mathrm{gr}} / x_{1}^{\mathrm{l}, \mathrm{bulk}}$ as a function of the relative undercooling $\Delta \theta$ for (a) the $\mathrm{Au} / \mathrm{Ni}$ mixture with $x_{1}^{1, \text { bulk }}=x_{\text {Au }}^{1, \text { bulk }}=0.4$ and b) the EEE-SSS mixture with $x_{1}^{1, \text { bulk }}=x_{\text {EEE }}^{1, \text { bulk }}=$ 0.8 , without transport limitations (dotted lines), with only mass transport limitation (dashed lines) for three values of $q_{\mathrm{m}}$, as indicated in the graphs, and with both mass and heat transport limitation (full lines) for the same values of $q_{\mathrm{m}}$ and with $q_{\mathrm{T}}^{1}=q_{\mathrm{m}} / 100$ for the $\mathrm{Au} / \mathrm{Ni}$ system and $q_{\mathrm{T}}^{1}=q_{\mathrm{m}} / 10$ for the EEE-SSS system.
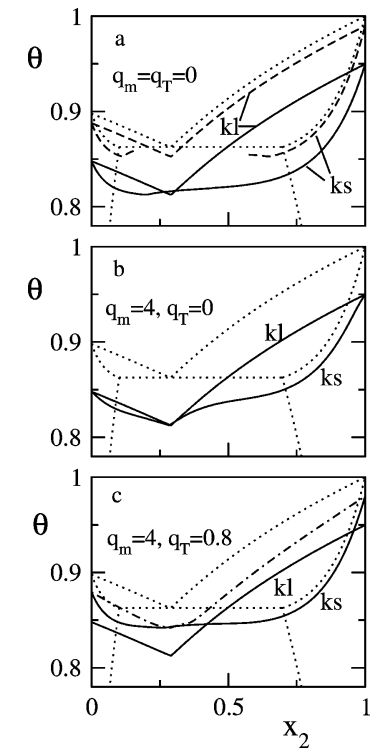

Figure 7. Effective kinetic phase diagrams for the eutectic system para-dichlorobenzene (1) and para-bromoiodobenzene (2) for relative undercoolings of 0.05 (full lines) and 0.01 (dashed line in graph a) with and without including mass and heat transport limitation, as indicated in the graphs. The dotted lines give the equilibrium phase diagram. The dashed-dotted line in graphs (c) give the temperature at the surface as a function of the liquid bulk composition. The used thermodynamic data, mostly taken from ref 36, are $\Delta H_{1,0} / R T_{2}=6.6497$, $\Delta H_{2,0} / R T_{2}=6.2396, g_{0}^{\mathrm{s}, \mathrm{exc}} / R T_{\mathrm{a} v}=1.75, g_{1}^{\mathrm{s}, \mathrm{exc}} / R T_{\mathrm{a} v}=0.65$, and $c_{\mathrm{p}, 1}^{1} / R$ $=c_{\mathrm{p}, 2}^{1} / R=20$. Here $T_{a v}=\left(T_{1}+T_{2}\right) / 2$. The kinetic liquidus and solidus are indicated as $\mathrm{kl}$ and $\mathrm{k} s$ respectively.

Finally, we have considered the eutectic system para-dichlorobenzene/para-bromoiodobenzene, using the thermodynamic data from ref 36. EKPDs for this system are shown in Figure 7 for a bulk undercooling of $\Delta \theta=0.05$. In addition, in Figure 7a we have drawn the EKPD for the relatively small undercooling of $\Delta \theta=0.01$ without including transport limitations (dashed lines). For this small undercooling $\Delta \theta=0.01$ the kinetic phase diagram still shows two disconnected solid phase branches, giving rise to a kinetic miscibility gap. For liquid compositions well left of the eutectic composition only a para-dichlorobenzene rich solid phase is growing with a composition according to the left solidus branch, whereas for liquid compositions well 
right of the eutectic composition only a para-bromoiodobenzenerich solid phase is growing according to the right branch. For bulk liquid compositions close to the eutectic composition (i.e., close to the kink in kinetic liquidus), there are three steadystate solutions according to the stability criterion (eq 12), of which only two are stable, one belonging to the left and one to the right branch. For $\Delta \theta=0.05$, the miscibilty gap has disappeared, implying that all solid phase compositions are kinetically accessible. Taking into account mass transport limitation further reduces the segregation as shown in Figure 7b. Including also heat transport limitation (Figure 7c) again leads to stronger segregation, as the surface temperature in this case is considerable higher, i.e., closer to equilibrium, than the liquid bulk temperature. We note that in Figures $7 b$ and $7 c$, to avoid too many lines we have not drawn the surface liquid composition line, which is between the kinetic liquidus and the equilibrium liquidus. In fact, this line is not required to read the effective segregation. In Figure $7 \mathrm{c}$ the growth composition for the given bulk liquid undercooling $\Delta \theta=0.05$ and a given bulk liquid composition is simply read as the intersection of the horizontal line crossing the dashed-dotted line at that liquid composition and the kinetic solidus.

\section{Summary and Discussion}

We have formulated a concise description of the effective segregation taking place during the crystallization of solid solutions from a binary liquid mixture which incorporates the interfacial segregation and both mass and heat transport limitations in a coupled way. The model, denoted as the linear effective kinetic segregation (LEKS) model, is based on nonequilibrium thermodynamics, yielding a linear growth rate for each component, and the theory of hydrodyamics with moving boundaries, assuming fixed boundary layers for both mass and heat transport. It allows for the construction of effective kinetic phase diagrams (EKPDs). In these diagrams, the composition of the solid phase that is growing at nonequilibrium conditions for a given undercooling $\Delta T$ of the bulk liquid phase is given as a function of the bulk liquid composition. Also the temperature and composition of the liquid phase at the interface can be read from these diagrams. By construction, for $\Delta T \rightarrow 0$, the EKPD according to the LEKS model becomes equal to the equilibrium phase diagram, as it should be.

We have calculated EKPDs for different types of systems, based on realistic values of the model parameters, including thermodynamic and transport properties and typical values for the growth rates and boundary layer widths. According to the LEKS model, the relative magnitude of the various processes affecting the kinetic segregation is quite different for different materials. For atomic systems with a small dissolution entropy $\Delta S_{\mathrm{m}}=\Delta H_{\mathrm{m}} / T_{\mathrm{m}}$, such as metal alloys, mass transport limitation is the most important factor reducing the segregration. For molecular systems with a relatively large $\Delta S_{\mathrm{m}}$ the interfacial undercooling plays an important role, giving rize to a significant reduction in the segregation already at modest undercoolings occurring at normal conditions in experiments. Whereas the effect of mass transport limitation on the segregation is weaker than for metal systems, heat transport is relatively slow for molecular systems, giving rize to a significant contribution to the effective segregation. For semiconductor systems, the effects of interfacial undercooling and mass transport are competing, whereas the effect of heat transport limitation on the effective segregation is almost negligible as for metal systems.

The LEKS model is based on a linear growth rate for each component. For growth at nonroughened faces however, the growth rate may be dominated by 2-dimensional (2-d) nucleation or by spiral dislocation(s) obeying different, nonlinear growth laws. Nonlinear growth laws can particularly occur for molecular systems, which usually grow below their roughening temperatures, implying a (large) barrier for 2-d nucleation. From lattice MC simulations of pure systems it was found that the growth rate is roughly proportional to the average number of kink sites per unit of surface area, $N_{\mathrm{k}}$, at the growth surface. Extending this to mixed systems one could write

$$
v=\hat{v} N_{\mathrm{k}}\left(\tilde{\sigma}^{\mathrm{surf}}\right) \tilde{\sigma}^{\mathrm{surf}}
$$

where $N_{\mathrm{k}}\left(\tilde{\sigma}^{\text {surf }}\right)$ accounts for the possible nonlinearity. The dependence of $N_{\mathrm{k}}$ on $\tilde{\sigma}^{\text {surf }}$ varies with the growth mechanism. In this approach the interfacial segregation, as given by eq 13, does not change since the prefactor $N_{\mathrm{k}}$ appearing in the numerator and the denominator cancels, but the coupling with transport processes as a function of the undercooling changes. Nevertheless, it will not change the trend in the EKPDs, and the relative contributions from mass and heat transport limitations to the effective segregation. In practice one may construct $N_{\mathrm{k}}\left(\sigma^{\text {surf }}\right)$ as a function of the experimentally applied bulk undercooling such that the calculated velocities match with the experimental velocities. Once this has been done, the EKPD values can be calculated.

On a microscopic level, $N_{\mathrm{k}}\left(\sigma^{\text {surf }}\right)$ depends of the surface kinetics, which depend on the crystallographic orientation of the crystal face. Currently, we have started studying this by means of lattice MC simulations of crystal growth for an arbitrary crystal structure, as implemented in the software program MONTY, ${ }^{39}$ but generalized to deal with solid solution growth.

Acknowledgment. This work has been sponsored by Stichting Technische Wetenschappen (STW), The Netherlands. We thank Dr. Kees van Miltenburg for his stimulating comments in many discussions.

\section{References and Notes}

(1) Kitaigorodskii, A. I. Mixed Crystals, Springer-Verlag: Berlin, 1984.

(2) van Eikeren, P. In Chiral separations: applications and technology; Ahuja, S., Ed.; American Chemical Society: Washington, DC, 1997; Chapter 2.

(3) Guggenheim, E. A. Thermodynamics, 5th ed.; North-Holland: Amsterdam, 1967

(4) Wallace, D. C. Thermodynamics of Crystals; Wiley: New York, 1972 .

(5) Lupis, C. H. P. Chemical Thermodynamics of Materials; NorthHolland: New York, 1983.

(6) Los, J. H.; van Enckevort, W. J. P.; Vlieg E.; Flöter, E. J. Phys. Chem. B 2002, 106, 7321.

(7) Los, J. H.; van Enckevort, W. J. P.; Vlieg, E.; Flöter, E.; Gandolfo, F. G. J. Phys. Chem. B 2002, 106, 7331.

(8) Cherepanova, T. A.; van der Eerden, J. P.; Bennema, P. J. Cryst. Growth 1978, 44, 537 399

(9) Cherepanova, T. A.; Dzelme, J. B. Cryst. Res. Technol. 1981, 16,

(10) Gilmer, G. H.; Bennema, P. J. Cryst. Growth 1972, 13, 148

(11) Gilmer, G. H.; Bennema, P. J. Appl. Phys. 1972, 43, 1347.

(12) Pfeiffer, H.; Klupsch, Th.; Haubenreisser W. Microscopic Theory of Crystal Growth; Akademic Verlag: Berlin, 1989.

(13) Rudolph, P. Mater. Sci. Forum 1998, 276, 1.

(14) Burton, J. A.; Prim, R. C.; Slichter, W. P. J. Chem Phys. 1953, 21 1987.

(15) Aziz, M. J. J. Appl. Phys. 1982, 53, 1158

(16) Aziz, M. J. J. Appl. Phys. Lett. 1983, 43, 552

(17) Jackson, K. A.; Gilmer, G. H.; Temkin, D. E. Phys. Rev. Lett. 1995 $75,2530$.

(18) Jackson, K. A.; Beatty, K. M.; Gudgel, K. A. J. Cryst. Growth 2004, 271, 481 .

(19) Beatty, K. M.; Jackson, K. A. J. Cryst. Growth 1997, 174, 28. 
(20) Campisano, S. U.; Floti, G.; Baeri, P.; Grimaldi, M. G.; Rimini, E. Appl. Phys. Lett. 1980, 37, 719.

(21) Baeri, P.; Poate, J. M.; Campisano, S. U.; Floti, G.; Rimini, E.; Cullis, A. G. Appl. Phys. Lett. 1980, 37, 912.

(22) White, C. W.; Wilson, S. R.; Appleton, B. R.; Young, F. W., Jr. J. Appl. Phys. 1980, 51, 738.

(23) Baeri, P.; Floti, G.; Poate, J. M.; Campisano, S. U.; Cullis, A. G. Appl. Phys. Lett. 1981, 38, 800 .

(24) Reitano, R.; Smith, P. M.; Aziz, M. J. J. Appl. Phys. 1994, 76, 2359.

(25) Kittl, J. A.; Aziz, M. J.; Brunco, D. P.; Thompson, M. O. J. Cryst. Growth 1995, 148, 172

(26) Matsumoto, N.; Kitamura, M. J. Cryst. Growth 2001, 222, 667.

(27) Matsumoto, N.; Kitamura, M. J. Cryst. Growth 2002, 237, 51.

(28) Kitamura, M.; Matsumoto, N. J. Cryst. Growth 2004, 260, 243

(29) Los, J. H.; Flöter, E. J. Phys. Chem. Chem. Phys. 1999, 1, 4251.

(30) Los, J. H.; van Enckevort, W. J. P., Vlieg, E., work in progress.

(31) Redlich, O.; Kister, A. T. Ind. Eng. Chem. 1948, 40, 345.
(32) Oonk, H. A. J.; Mondieig, D.; Haget, Y.; Cueveas-Diarte, M. A. J. Chem. Phys. 1998, 108, 715 .

(33) van der Kemp, W. J. M.; Blok, J. G.; van der Linde, P. R.; Oonk, H. A. J.; Schuijff, A.; Verdonk, M. L. Thermochim. Acta 1993, 225, 17. (34) Rosenberger, F. Fundamentals of Crystal Growth I; Springer Series in Solid-State Sciences 5; Cardona, M., Fulde P., Queisser, H. J., Eds.; Springer-Verlag: Berlin, 1997.

(35) Ogando Arregui, E.; Caro, M.; Caro, A. Phys. Rev. B 2002, 66 , 054201.

(36) Oonk, H. A. J.; Calvet, T.; Cuevas-Diarte, M. A.; Tauler, E.; Labrador, M.; Haget, Y. Thermochim. Acta 1995, 250, 13-18.

(37) van der Linde, P. R.; Bolech, M.; den Besten, R.; Verdonk, L. M.; Miltenburg, J. C.; Oonk, H. A. J. J. Chem. Thermodyn. 2002, 34, 613.

(38) Wesdorp, L. H. Liquid-multiple solid-phase equilibria in fats; Ph.D. Thesis, University Delft, The Netherlands, 1990.

(39) Boerrigter, S. X. M.; Josten, G. P. H.; van de Streek, J.; Hollander, F. F. A.; Los, J. H.; Cuppen, H. M.; Bennema, P.; Meekes, H. J. Phys. Chem. A 2004, 108, 5894. 